\title{
Histórico familiar para câncer de mama em mulheres: estudo populacional em Uberaba (MG) utilizando o Family History Screen-7
}

\author{
Family history of breast cancer in women: a population-based study \\ in Uberaba (MG) using the Family History Screen-7
}

\author{
Mariana Colombini Buranello', Isabel Aparecida Porcatti de Walsh², Gilberto de Araújo Pereira ${ }^{\mathbf{3}}$ \\ Shamyr Sulyvan de Castro ${ }^{4}$
}

DOI: 10.1590/0103-1104202113009

\footnotetext{
1 Universidade de Franca (Unifran) - Franca (SP), Brasil.

mburanello@unifran.edu.br

2 Universidade Federal do Triângulo Mineiro (UFTM), Instituto de Ciências da Saúde (ICS), Departamento de Fisioterapia Aplicada (DFA) - Uberaba (MG), Brasil.

3 Universidade Federal do Triângulo Mineiro (UFTM), Instituto de Ciências da Saúde (ICS), Departamento de Ensino da Enfermagem e Educação Comunitária (DEEEC) - Uberaba (MG), Brasil.

4 Universidade Federal do Ceará (UFC), Programa de Pós-Graduação em Saúde Pública (PPGSP) Fortaleza (CE), Brasil.
}

\begin{abstract}
RESUMO Objetiva-se determinar a prevalência de mulheres com risco de desenvolvimento de câncer de mama pelo histórico familiar em Uberaba (MG), e essa prevalência nas diferentes condições socioeconômicas e epidemiológicas. Pesquisa quantitativa, analítica, observacional e transversal de base populacional. Amostra com 1.520 mulheres a partir de 20 anos. Dados coletados por entrevistas domiciliares sobre informações socioeconômicas e epidemiológicas. A avaliação do risco hereditário de câncer de mama foi realizada pelo Family History Screen-7 (FHS-7). Como resultado, 28,6\% da amostra apresentaram risco hereditário para câncer de mama. Houve associação significativa do risco de câncer quanto a: faixa etária, sendo nas mulheres com idade de 70 anos ou mais a maior proporção com risco (33,5\%); etnia, sendo a maior proporção entre mulheres de etnia branca (31,2\%); renda familiar, com maior ocorrência para renda per capita > 2,5 salários-mínimos (31,6\%); prática de exames preventivos (30,6\%); e alterações benignas na mama (46,7\%). Conclui-se que, a partir do histórico familiar pelo FHS-7, o risco de as mulheres desenvolverem câncer mostrou-se significativamente associado com maior faixa etária, etnia branca e maior renda per capita, com a prática de exames preventivos em dia e alterações benignas na mama. Tais resultados possibilitam melhores direcionamentos de políticas públicas.
\end{abstract}

PALAVRAS-CHAVE Saúde da mulher. Neoplasias da mama. Fatores de risco. Inquéritos epidemiológicos. 


\section{Introdução}

Mundialmente, o tipo de câncer mais incidente nas mulheres é o de mama, correspondendo a 25,2\% do total de novos casos. Já nos homens, é mais raro, com um caso para cada 100 casos femininos ${ }^{1}$. De acordo com o Instituto Nacional de Câncer (Inca), no Brasil, estimativas do biênio 2018/2019 demonstram que a incidência dessa doença foi de, aproximadamente, 59 mil novos casos $^{2}$. Para 2020, estimam-se cerca de 66.280 novos $\operatorname{casos}^{3}$, demonstrando o crescimento da incidência desse tipo de câncer no País. Os principais fatores de risco para o desenvolvimento do câncer de mama na mulher estão ligados a fatores endócrinos, genético-hereditários, aos hábitos de vida e ao envelhecimento ${ }^{4}$.

Sobre os fatores genético-hereditários, de acordo com o Inca, cerca de 5 a 10\% do total de casos são de caráter hereditário 5 . $\mathrm{O}$ risco hereditário envolve mutações genéticas relacionadas, principalmente, aos genes BRCAl e 2 . Além desses genes, outros já são relacionados ao desenvolvimento do câncer de mama. Os genes BRCA1 e BRCA2 são supressores de tumor, sendo que, em indivíduos com risco hereditário, há a herança de determinada mutação em um dos seus alelos, predispondo à sua inativação e ao consequente desenvolvimento do carcinoma. Além desses genes, outras mutações genéticas de síndromes multicânceres, que incluem o câncer de mama, já foram identificadas, como o TP53, PTEN, $\mathrm{MLH}$, entre outros ${ }^{6}$. O acesso à testagem genética é muito restrito, ficando reservado a grandes centros de pesquisa.

O Sistema Único de Saúde (SUS) não cobre a realização do teste, e, no âmbito da saúde suplementar, a Nota Técnica da Agência Nacional de Saúde Suplementar (ANS) nº 876/2013 determinou a cobertura de diversas testagens genéticas, incluindo BRCAl e $2^{7}$. Visto que a testagem genética é um procedimento caro e restrito, deve-se atentar para as características epidemiológicas de histórico familiar da população para identificação das mulheres em risco para início das estratégias de rastreamento ${ }^{8}$.
A história familiar, principalmente em parentes de primeiro grau, é considerada um importante fator de risco para o câncer de mama antes dos 50 anos $^{6}$. Consideram-se como em risco hereditário para câncer de mama mulheres que possuem vários casos de câncer de mama e/ou pelo menos um caso de câncer de ovário em parentes consanguíneos, sobretudo em idade jovem. Homens que possuam casos envolvendo parentes consanguíneos também podem ter predisposição genética, e são consideradas de maior risco para a doença ${ }^{8}$.

De acordo com os critérios do Inca, as mulheres consideradas com risco elevado para o desenvolvimento do câncer de mama são aquelas que apresentam: história familiar de câncer de mama em parente de primeiro grau antes dos 50 anos ou de câncer bilateral ou de ovário em qualquer idade; história familiar de câncer de mama masculino; e diagnóstico histopatológico de lesão mamária proliferativa com atipia ou neoplasia lobular in situ 9 .

No entanto, a informação sobre o histórico familiar de câncer é pouco explorada, ficando reservada a consultas especializadas. No que diz respeito à atenção básica, essa informação é muitas vezes negligenciada, impedindo a detecção de indivíduos com risco hereditário, bem como seu encaminhamento para serviços especializados. Uma alternativa para abordar a questão familiar nos serviços primários de saúde é a utilização de ferramentas para facilitar a detecção da necessidade de rastreamento em mulheres com histórico familiar de câncer. O instrumento Family History Screen 7 (FHS-7) foi desenvolvido no Brasil com o objetivo de ser uma ferramenta de indicação do risco hereditário para desenvolvimento do câncer de mama ${ }^{10}$.

Dessa forma, traçar um perfil das mulheres classificadas com o risco familiar pode trazer mais conteúdo para a formulação de estratégias de abrangência a essa população de provável risco, com enfoque na prevenção da doença. Assim, a presente pesquisa tem por objetivo determinar a prevalência geral de mulheres com risco de desenvolvimento 
de câncer de mama pelo histórico familiar no município de Uberaba (MG), bem como essa prevalência nas diferentes condições socioeconômicas e epidemiológicas.

\section{Material e métodos}

Pesquisa de caráter quantitativo, analítico, observacional e transversal, de base populacional. Este estudo é parte de um projeto maior - Inquérito de Saúde da Mulher em Uberaba-MG (ISA Mulher Uberaba-MG) -, aprovado pelo Comitê de Ética em Pesquisa (1826/2010). O projeto ISA Mulher Uberaba tem por objetivo geral conhecer o perfil de saúde das mulheres a partir de 18 anos na cidade de Uberaba, MG. A cidade de Uberaba localiza-se no estado de Minas Gerais, na região do triângulo mineiro, com população de 295.988 habitantes, densidade demográfica de $65,5 \mathrm{hab} / \mathrm{km}^{2}$, sendo a maior cidade da macrorregião a que pertence ${ }^{11}$.

Sobre os aspectos éticos, foram apresentados aos sujeitos os objetivos da pesquisa, as informações pertinentes sobre ela e o Termo de Consentimento Livre e Esclarecido (TCLE). Somente após a assinatura do TCLE foi conduzida a entrevista.

A amostra do estudo foi composta por mulheres a partir de 20 anos residentes na cidade de Uberaba (MG), Brasil. Os critérios de inclusão foram: mulheres residentes na zona urbana da cidade de Uberaba, com idade a partir de 20 anos, que compreenderam o teor da pesquisa e estavam aptas a responder à entrevista após assinatura do Termo de Consentimento Livre e Esclarecido.

Para o cálculo amostral, foi considerada uma amostra com 1.530 mulheres, mais $20 \%$ de perda. Essa quantidade (1.530) foi calculada considerando-se: a ausência de conhecimento a priori sobre as estimativas das prevalências dos eventos de interesse, portanto, todos iguais a $50 \%$; a necessidade de inferir as estimativas de interesse encontradas na amostra sobre a população considerando um nível de confiança de $95 \%$ e margem de erro de no máximo $2,5 \%$. O tamanho amostral final foi de 1.836 .

Para que a amostra tivesse representatividade quanto aos estratos faixa de idade, renda, escolaridade e cor da pele, ela foi composta com as mesmas proporções populacionais desses estratos, em especial, quanto à faixa de idade. Os dados do Censo do IBGE" (Instituto Brasileiro de Geografia e Estatística) do ano de 2010 foram utilizados como base para esse plano amostral. Do total de 1.560 entrevistas realizadas no ISA Mulher Uberaba no ano de 2014, 1.520 correspondem às mulheres com 20 anos ou mais, compondo a amostra da presente pesquisa, desta forma, 40 mulheres com menos de 20 anos foram excluídas da análise. Foram incluídas mulheres a partir de 20 anos, haja vista que levou-se em consideração a análise de mulheres consideradas adultas pela Organização Mundial da Saúde $(\mathrm{OMS})^{\mathbf{1 2}}$, além de as recomendações do Inca relatarem ações de rastreio para o câncer de mama a partir dos 20 anos $^{13}$.

Para a seleção da amostra, foi considerada uma amostragem probabilística em múltiplos estágios. No primeiro estágio, dentro de cada distrito sanitário, foram selecionados aleatoriamente $50 \%$ dos bairros, respeitando-se a proporcionalidade populacional de cada distrito em termos de quantidade de bairros. No segundo estágio, dentro de cada bairro previamente sorteado, foram selecionados, de forma aleatória, $25 \%$ dos setores censitários, respeitando-se a proporcionalidade populacional de cada bairro em termos de quantidade de setores censitários. Dentro do setor censitário previamente sorteado, os domicílios foram selecionados de forma sistemática a partir da seleção aleatória do primeiro domicílio, e os demais foram aqueles na sequência entre 32 domicílios, até ser atingida a quantidade desejada para cada estrato de interesse quanto à faixa de idade. Apenas uma entrevista foi realizada para cada domicílio selecionado, sendo que, no caso de haver mais de uma mulher no mesmo, a 
escolha sobre a realização da entrevista ficou a critério das moradoras.

A avaliação do risco hereditário de câncer de mama foi realizada através de um questionário validado para a população brasileira, o Family History Screen 7 (FHS-7) ${ }^{\mathbf{1 0}}$, proposto como um instrumento de rastreio para o risco hereditário de desenvolvimento do câncer de mama em um contexto de atenção básica. Esse questionário é composto de sete perguntas com respostas afirmativas ou negativas. $\mathrm{O}$ ponto de corte utilizado foi de uma resposta afirmativa revelando o risco hereditário para o desenvolvimento do câncer de mama. Para esse ponto de corte, os autores do questionário verificaram uma sensibilidade de $87,6 \%$ e especificidade de $56,4 \%{ }^{9}$. O questionário utilizado foi selecionado por ser um instrumento validado à população brasileira ${ }^{10}$.

A coleta dos dados foi realizada por meio de entrevistas domiciliares, feitas por 22 entrevistadoras do sexo feminino, especialmente treinadas para este estudo. Foram dadas as orientações quanto ao funcionamento dos instrumentos. Cada uma das entrevistadoras passou por treinamento de aplicação do questionário, além de noções e técnicas para entrevista. Também houve a supervisão de campo com conferência das entrevistas no recebimento, e a checagem aleatória via telefone de $10 \%$ das entrevistas realizadas. O período de coleta dos dados ocorreu entre os meses de março a outubro de 2014.

Para traçar o perfil socioeconômico, epidemiológico do grupo, as variáveis foram: faixa etária $(20 \nmid 39 ; 40 \dashv 49 ; 50 \dashv 69 ; \geq 70)$; cor da pele (branca; parda; preta; outras); estado conjugal (solteiro; em união; viúva; separada); escolaridade (analfabeta; até 5 anos; $>5 \mathrm{a}<9$ anos; $9 \mathrm{a}<12 ; 12$ anos ou mais); renda familiar per capita (em Salários-Mínimos - SM) (até 0,5 SM; > 0,5 - $1 \mathrm{SM}$; > $1 \mathrm{SM}$; > 1 a 2,5 SM; > 2,5 SM); chefe de família (sim; não); nuliparidade (sim; não); presença de neoplasias (sim; não); neoplasias benignas da mama (sim; não); câncer de mama (sim; não); prática de exames preventivos (sim; não). A prática de exames preventivos tomou como recomendação a mamografia bianual na faixa etária entre 50-69 anos, e o exame clínico das mamas como parte da rotina de atenção à saúde da mulher, de acordo com as recomendações do Inca ${ }^{13}$.

As variáveis para identificação do risco de câncer de mama pelo histórico familiar foram aquelas que compõem do questionário FHS710: Parentes de $1^{\circ}$ grau com câncer de mama ou de ovário (Sim/Não); Câncer de mama bilateral (Sim/Não); Homem da família com câncer de mama (Sim/Não); Mulher da família com câncer de mama ou de ovário (Sim/Não); Mulher da família com câncer de mama antes dos 50 anos (Sim/Não); Dois ou mais parentes com câncer de mama e/ou de ovário (Sim/Não); Dois ou mais parentes com câncer de mama e/ ou de intestino (Sim/Não). O ponto de corte de uma resposta afirmativa foi utilizado para determinar o risco pelo histórico familiar, conforme sugerido pelos autores do instrumento ${ }^{10}$.

Após a coleta das entrevistas, houve a revisão e a codificação dos dados. Foi construído um banco de dados eletrônico, no programa EpiData ${ }^{\circledR}$ versão2.0. Os dados coletados foram processados em microcomputador, com dupla digitação e verificação da consistência entre os dois bancos, e, na ocorrência de dados inconsistentes, estes foram verificados na entrevista original, e realizada sua correção.

A análise descritiva foi realizada com uso de frequências absolutas e percentuais. Os testes inferenciais usaram o teste de Qui-Quadrado com nível de significância de $\mathrm{p}<0,05$. $O$ teste qui-quadrado apresentou em todas as situações condições básicas para sua utilização, como, pelo menos, $80 \%$ das células com frequências maiores que 5 e nenhuma menor que $1^{\mathbf{1 4}}$. Todas as análises foram realizadas com o programa Stata $^{\circledR}$ versão 13.

\section{Resultados}

Segundo a classificação de risco proposta pelo FHS-7, entre 1.516 mulheres com risco de câncer avaliadas pelo instrumento FHS-7, 
28,6\% (IC95\%: 26,3\%-30,8\%) apresentam risco para o desenvolvimento do câncer de mama.

A análise do risco de câncer avaliado pelo FHS-7 segundo algumas variáveis sociodemográficas, demonstra uma associação significativa da presença do risco de câncer com: a faixa etária ( $p=0,001)$, sendo naquelas mulheres com idade de 70 anos ou mais a maior proporção de mulheres com risco de desenvolvimento de câncer de mama pelo histórico familiar - 33,5\% ( IC95\%: 27,9\%; 40,0\%); com a etnia ( $\mathrm{p}=0,01$ ), sendo a maior proporção entre as mulheres de etnia branca - 31,2\% ( IC95\%: 28,1\%; 34,4\%); com a renda familiar $(\mathrm{p}=0,022)$, havendo maior ocorrência entre as mulheres com renda per capita > 2,5 salários-mínimos - 31,6\% ( IC95\%: $24,9 \% ; 38,2 \%$ ) (tabela 1).

Quanto à análise de associação do risco de câncer avaliado pelo instrumento FHS-7 segundo algumas variáveis epidemiológicas, observa-se uma associação significativa quanto à prática de exames preventivos $(\mathrm{p}=0,024)$ (30,6\%; IC95\%: 27,7\%; 33,6\%) e alterações benignas na mama ( $\mathrm{p}=0,027)(46,7 \%$; IC95\%: $28,8 \% ; 64,5 \%$ ) (tabela 2 ).

A tabela 1 apresenta a caracterização sociodemográfica da amostra segundo o risco determinado pelo instrumento FHS-7.

Tabela 1. Caracterização sociodemográfica das mulheres em Uberaba, segundo o risco hereditário pelo FHS-7. ISA Mulher, Uberaba (MG), 2014

\begin{tabular}{|c|c|c|c|c|c|c|c|}
\hline \multirow[b]{2}{*}{ Variável } & \multicolumn{3}{|c|}{ Risco pelo FHS-7^ } & \multirow[b]{2}{*}{$p^{\star \star}$} & \multirow[b]{2}{*}{ Total N (\%) } & \multirow[b]{2}{*}{ IC $95 \%$} & \\
\hline & $\operatorname{Sim} n(\%)$ & IC $95 \%$ & Não n (\%) & & & & \\
\hline Geral & $433(28,6 \%)$ & $26,3-30,8$ & $1083(71,4 \%)$ & - & $1516(100 \%)$ & - & - \\
\hline Faixa Etária & $433(28,6 \%)$ & IC95\% & $1083(71,4 \%)$ & 0,001 & $1516(100 \%)$ & IC $95 \%$ & \\
\hline $20 \dashv 39$ anos & $103(22,5 \%)$ & $18,7-26,3$ & $355(77,5 \%)$ & & $458(30,2 \%)$ & 27,9 & 32,5 \\
\hline $40-49$ anos & $64(26,1 \%)$ & $20,6-31,6$ & $181(73,9 \%)$ & & $245(16,2 \%)$ & 14,3 & 18,0 \\
\hline $50-69$ anos & $198(32,5 \%)$ & $28,7-36,2$ & $412(67,5 \%)$ & & $610(40,2 \%)$ & 37,8 & 42,7 \\
\hline$\geq 70$ anos & $68(33,5 \%)$ & $27,9-40,0$ & $135(66,5 \%)$ & & $203(13,4 \%)$ & 11,7 & 15,1 \\
\hline Escolaridade $\mathrm{e}^{\star \star \star}$ & $433(28,6 \%)$ & IC95\% & $1083(71,4 \%)$ & 0,076 & $1516(100 \%)$ & IC 95\% & \\
\hline Analfabeta & $10(18,2 \%)$ & $8,0-28,4$ & $45(81,8 \%)$ & & $55(3,6 \%)$ & 2,7 & 4,6 \\
\hline Até 5 anos & $96(29,4 \%)$ & $24,5-34,4$ & $230(70,5 \%)$ & & $326(21,5 \%)$ & 19,4 & 23,6 \\
\hline$>5 \mathrm{a}<9$ anos & $87(24,5 \%)$ & $20,0-29,0$ & $268(75,5 \%)$ & & $355(23,4 \%)$ & 21,3 & 25,5 \\
\hline $9 a<12$ anos & 85 (29\%) & $23,8-34,2$ & $208(71 \%)$ & & $293(19,3 \%)$ & 17,3 & 21,3 \\
\hline 12 anos ou mais & $155(31,8 \%)$ & $27,7-36,0$ & $332(68,2 \%)$ & & $487(32,1 \%)$ & 29,8 & 34,5 \\
\hline Etnia & $431(28,6 \%)$ & IC95\% & $1076(71,4 \%)$ & 0,010 & $1507(100 \%)$ & IC 95\% & \\
\hline Branca & $263(31,2 \%)$ & $28,1-34,4$ & $579(68,8 \%)$ & & $842(55,9 \%)$ & 53,4 & 58,4 \\
\hline Preta/Parda & $162(26 \%)$ & $22,6-29,4$ & $461(74 \%)$ & & $623(41,3 \%)$ & 38,9 & 43,8 \\
\hline Outras & $6(14,3 \%)$ & $3,7-24,9$ & $36(85,7 \%)$ & & $42(2,8 \%)$ & 2,0 & 3,6 \\
\hline Chefe Família & $192(30 \%)$ & $26,6-33,8 \%$ & $448(70 \%)$ & 0,278 & $640(43,4 \%)$ & 40,8 & 45,9 \\
\hline 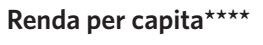 & $433(28,6 \%)$ & IC95\% & $1083(71,4)$ & 0,022 & $1516(100 \%)$ & IC 95\% & \\
\hline Até 0,5 SM & $84(22,3 \%)$ & $18,1-26,6$ & $292(77,7 \%)$ & & $376(24,8 \%)$ & 22,6 & 27,0 \\
\hline$>0,5-1 S M$ & $160(30,6 \%)$ & $26,6-34,5$ & $363(69,4 \%)$ & & $523(34,5 \%)$ & 32,1 & 36,9 \\
\hline$>1 \mathrm{SM}-2,5 \mathrm{SM}$ & $130(30,2 \%)$ & $25,9-34,6$ & $300(69,8 \%)$ & & $430(28,4 \%)$ & 26,1 & 30,6 \\
\hline$>2,5 \mathrm{SM}$ & $59(31,5 \%)$ & $24,9-38,2$ & $128(68,4 \%)$ & & $187(12,3 \%)$ & 10,7 & 14,0 \\
\hline
\end{tabular}

Fonte: Elaboração própria.

${ }^{\star}$ FHS-7: Family History Screen 7; ${ }^{\star \star}$ Teste de Qui-Quadrado; ${ }^{\star \star \star}$ Escolaridade em anos de estudo; ${ }^{\star \star \star \star}$ Renda per capita em Salários-Mínimos (SM); número máximo de perdas: 183. 
De acordo com a tabela 1, a faixa etária que demonstrou maior frequência de risco foi a de 70 anos ou mais, etnia branca, renda per capita $>$ 2,5 salários-mínimos.
A tabela 2 abrange as características epidemiológicas da amostra segundo o risco pelo instrumento FHS-7.

Tabela 2. Caracterização epidemiológica das mulheres em Uberaba, segundo o risco hereditário pelo FHS-7. ISA Mulher, Uberaba (MG), 2014

\begin{tabular}{lrrrrrrr}
\hline & \multicolumn{3}{c}{ Risco pelo FHS-7 } & & & \\
\cline { 2 - 5 } Variável & Sim n (\%) & IC 95\% & Não n (\%) & p ${ }^{\star \star}$ & Total N (\%) & IC 95\% \\
\hline Nuliparidade & $70(31 \%)$ & $24,9-37,0$ & $156(69 \%)$ & 0,394 & $226(15 \%)$ & $15,0-19,1$ \\
Prática de exames preventivos & $288(30,6 \%)$ & $27,7-33,6$ & $653(69,4 \%)$ & 0,024 & $941(62,4 \%)$ & $68,6-73,5$ \\
Alterações Benignas Mama & $14(46,7 \%)$ & $28,8-64,5$ & $16(53,3 \%)$ & 0,027 & & $30(2 \%)$ & $1,5-3,1$ \\
Câncer de mama & $4(57,1 \%)$ & $20,5-93,8$ & $3(42,9 \%)$ & 0,093 & $7(0,5 \%)$ & $0,1-0,9$ \\
Neoplasias Geral & $43(35,9 \%)$ & $27,3-44,4$ & $77(64,2 \%)$ & 0,068 & & $120(7,9 \%)$ & $7,5-10,6$ \\
\hline
\end{tabular}

Fonte: Elaboração própria.

*FHS-7: Family History Screen 7; **Qui-Quadrado; número máximo de perdas: 183.

A tabela 2 demonstra que mais de $30 \%$ das mulheres que praticam os exames preventivos para câncer de mama apresentaram o risco hereditário segundo o FHS-7. Além disso, o risco familiar positivo esteve presente em $46,67 \%$ dos casos de alterações benignas na mama. Dos casos de câncer de mama prevalentes na amostra, mais da metade $(57,1 \%)$ revelou histórico familiar positivo pelo FHS-7.

Sobre a prática de exames preventivos para câncer de mama (mamografia), 66,5\% das mulheres classificadas com o risco familiar pelo FHS-7 estavam em dia com o exame. Esse valor foi menor para as que não estavam classificadas com o risco familiar (60,9\%).

\section{Discussão}

Sabe-se que existe um vasto potencial de ações de prevenção primária e secundária do câncer de mama não realizadas em populações de risco de desenvolvimento dessa doença ${ }^{15}$. Ainda, o histórico familiar de câncer aumenta o risco de desenvolvimento de vários tipos de câncer, em especial, o de mama 5 .
Na presente pesquisa foi encontrada uma prevalência do risco familiar para o câncer de mama de 28,6\%, segundo o instrumento FHS-7. No estudo de Renck et al. ${ }^{16}$, foi relatada uma prevalência semelhante (22\%).

Caleffi et al. ${ }^{17}$ conduziram um estudo de coorte prospectiva com mais de 3 mil mulheres em Porto Alegre, capital do Rio Grande do Sul, Brasil, avaliando-se a aderência a programas de rastreamento para o câncer de mama e fatores associados. Os autores também utilizaram o FHS-7 para avaliação do risco familiar do câncer de mama, e as mulheres com suspeita de síndrome hereditária de câncer de mama foram encaminhadas para o serviço de aconselhamento genético. Os resultados da pesquisa demonstraram, inicialmente, uma prevalência de $33,5 \%$ de histórico familiar de câncer em parentes de primeiro grau, 5,3\% de histórico familiar de câncer de mama ou ovário em parentes de primeiro grau, e, destes, 3,3\% apresentaram a mutação genética. Os autores do artigo destacam a importância da comunicação adequada entre os serviços de saúde e as mulheres que apresentaram histórico familiar positivo, pois estas necessitam de 
programas de rastreamento adequados, o que pode facilitar sua aderência quando a mulher possui a informação sobre esse risco familiar ${ }^{17}$.

O estudo de Cloud et al. ${ }^{15}$ avaliou uma coorte de mulheres de alto risco para desenvolvimento do câncer de mama nos Estados Unidos, observando uma maior prevalência de mulheres brancas, com nível educacional médio e superior e idade entre 46 e 55 anos. No estudo de Bellcross et al. ${ }^{18}$, foi constatada maior prevalência da etnia branca, escolaridade média, maior nível de renda e idade entre 51-60 anos para um grupo de mulheres encaminhadas para aconselhamento genético e testagem dos genes BRCAl/2.

Na presente pesquisa, foi encontrada maior prevalência do risco familiar pelo FHS-7 na etnia branca, com maior renda per capita e na faixa etária de 70 anos ou mais. Os resultados referentes a etnia concordam com os dos estudos de Cloud et al. ${ }^{15}$ e Bellcross et al. ${ }^{18}$. Sabe-se que a etnia branca apresenta maior prevalência de mutação no gene BRCAl em comparação à negra ${ }^{19}$.

Os achados sobre a renda refletem um perfil socioeconômico da amostra classificada com risco familiar pelo FHS-7. Blaes et al. ${ }^{20}$ relataram maior renda para mulheres com testagem genética positiva para o câncer de mama, o que pode refletir a relação entre renda e maior capacidade de acesso à testagem genética. No entanto, na presente pesquisa, tal análise não foi controlada por outras variáveis que poderiam influenciar esse quadro, como etnia e escolaridade, podendo ser considerada uma limitação da presente pesquisa. No estudo de Bellcross et al. ${ }^{18}$, a renda deixou de ser associada ao risco familiar após ser controlada por outras variáveis.

Sobre o perfil da faixa etária mais avançada encontrado na presente pesquisa, apesar da história familiar de câncer de mama ser reconhecida como preditor de risco para cânceres de início precoce, não são observadas variações nessa relação de risco em idades mais avançadas. Assim, essa característica permanece como risco mesmo com o envelhecimento ${ }^{19,21}$.
Sabe-se que o rastreamento mamográfico para o câncer de mama é a melhor metodologia de prevenção secundária em nível populacional, constituindo medida de intervenção, promovendo a detecção precoce na fase assintomática e implicando a redução substancial da morbimortalidade causada pelo diagnóstico tardio 22 . Ao se analisar a prática dos exames preventivos, $66,51 \%$ das mulheres classificadas com o risco familiar pelo FHS-7 estavam em dia com esse tipo de rastreamento, correspondendo a aproximadamente $30 \%$ do total de mulheres que estavam em dia com essa prática. Esse resultado indica que o histórico familiar da doença pode auxiliar a adesão às práticas de rastreio, já que o histórico familiar da doença está associado, também, a maior frequência de conhecimento dos fatores de risco ${ }^{23}$. No estudo de Brum et al. ${ }^{24}$, foi observada uma prevalência de $35,5 \%$ de mulheres atendidas em um ambulatório de um hospital universitário com relato de familiares com câncer de mama, e encontrou-se uma associação entre ter familiares com câncer de mama em específico e a adesão às práticas de rastreamento (mamografia) e de exames de atenção à saúde das mamas (exame clínico e autopalpação). Os autores justificam tais achados ressaltando que conhecer alguém com câncer de mama ou, ainda mais fortemente, algum familiar com a doença pode levar a mulher a ter mais consciência sobre a doença e ser proativa no processo de autocuidado 24 .

Mais de $46 \%$ das mulheres que relataram alterações benignas nas mamas foram classificadas com o risco familiar pelo FHS-7. O resultado é um alerta à necessidade de abrangência das ações de atenção a esse grupo, em específico, com necessidade de aprofundamento nas questões de pesquisa de câncer familiar e aprofundamento diagnóstico de casos suspeitos $^{\mathbf{1 3}}$. As alterações benignas da mama associadas ao risco familiar da doença devem ser investigadas, pois são um fator de risco ao desenvolvimento do câncer de mama ${ }^{8}$.

As diretrizes para diagnóstico precoce do câncer de mama do Inca indicam a importância 
de se fazer o acompanhamento e encaminhamento de casos suspeitos na atenção básica, ressaltando a importância das estratégias de conscientização e do exame clínico das mamas como parte desse cuidado à saúde da mulher ${ }^{13}$. Nesse sentido, o instrumento FHS-7 é proposto como uma forma de rastreio para o risco familiar do câncer de mama, buscando a detecção desses casos de forma mais rápida em um contexto de atenção básica ${ }^{10}$, sendo indicado como uma das ferramentas de rastreamento segundo recomendações da United States Preventive Services Task Force (USPSTS) ${ }^{\mathbf{2 5}}$. De acordo com o documento, a classificação de risco pelo FHS-7 deve ser utilizada como um rastreamento para a necessidade de encaminhamento para testagem genética de $\mathrm{BRCAl} / \mathbf{2}^{\mathbf{2 4}}$. Assim, não é possível afirmar que todas as mulheres classificadas com o risco familiar pelo instrumento realmente sejam classificadas como o grupo de risco para o câncer de mama de fator hereditário ou familiar.

No âmbito do SUS, a testagem genética para determinação do risco familiar de desenvolvimento do câncer de mama não é uma realidade. A realização de testagem está restrita a centros de pesquisa especializados, e poucas mulheres terão acesso a esse tipo de exame ${ }^{8}$. A ANS já tem publicada uma nota técnica que determina a realização da testagem, após o preenchimento de diversas características que evidenciam a possibilidade da genética familiar para diversos tipos de doenças ${ }^{7}$. Dessa forma, as estratégias de rastreio eficazes, avaliação do histórico familiar para câncer em consultas de rotina na atenção básica, educação das mulheres com relação aos sinais/sintomas do câncer de mama e hábitos de vida saudáveis são a base para o controle e o segmento das mulheres detectadas com possível risco familiar para o desenvolvimento do câncer de mama, com consultas mais frequentes, exames de rastreamento com periodicidade especial e o autocuidado ${ }^{5,8}$.

Considerando que a informação sobre o histórico familiar de câncer é pouco explorada e muitas vezes negligenciada na atenção básica, e que há diversos fatores ou barreiras que podem limitar a efetividade do rastreamento mamográfico, quer estejam relacionados ao sistema de saúde, à educação ou à adesão ao exame de mamografia ${ }^{22}$, esse instrumento é indicado como uma ferramenta de fácil aplicação na atenção básica para o rastreamento inicial de mulheres classificadas com o risco familiar para posteriores análises específicas desse risco familiar ${ }^{\mathbf{2 6}}$, podendo trazer mais conteúdo para a formulação de estratégias de abrangência a essa população de provável risco.

No entanto, cabe considerar que, apesar da sua importante indicação no rastreamento de mulheres com risco familiar de câncer de mama, existem algumas limitações na realização de um questionário como o FHS-7. Uma delas é o viés de memória. Muitas mulheres podem não se lembrar, no momento da entrevista, da ocorrência de casos de câncer na família ou podem não conhecer a história de doenças da família e acabar por pressupor a ocorrência ou não da doença.

Outra questão é o ponto de corte de uma resposta afirmativa para classificação do risco familiar, além de todas as questões sobre esse histórico apresentarem o mesmo peso para a classificação. Esse ponto de corte foi proposto pelos autores do instrumento ${ }^{9}$, no entanto, não foram testados outros pontos de corte com uma especificidade maior. Como implicações para uma baixa especificidade existe a possibilidade da seleção de mulheres que não apresentam os marcadores genéticos para a testagem e o acompanhamento, gerando uma sobrecarga nos sistemas de saúde, além dos efeitos individuais de ansiedade e sobrecarga com a possibilidade do diagnóstico.

Como limitações do estudo, pode-se destacar o viés de memória a que inquéritos desse tipo estão sujeitos, visto que as participantes podem não se lembrar ou não conhecer o seu histórico familiar para algumas condições. Além disso, não foi realizado o controle de algumas variáveis para as medidas de associação. 


\section{Conclusões}

De forma geral, de acordo com os resultados da presente pesquisa, pode-se afirmar que o risco pelo histórico familiar determinado pelo FHS-7 demonstrou uma prevalência maior de mulheres da etnia branca, renda per capita maior e idade mais avançada na amostra estudada. A prevalência do risco familiar de câncer de mama pelo FHS-7 na amostra geral foi de $28,6 \%$, assim, tais resultados possibilitam a formulação de políticas públicas para prevenção e diagnóstico precoce do câncer de mama para essa população de risco, além de ressaltarem a importância da abordagem do histórico familiar de câncer de mama no contexto da atenção básica.

\section{Referências}

1. Torre LA, Freddie B, Siegel RL, et al. A Global Cancer Statistics, 2012. CA Cancer J. Clin. 2015; 65(2):87-108.

2. Instituto Nacional de Câncer José Alencar Gomes da Silva, Coordenação de Prevenção e Vigilância. Estimativa 2017: Incidência de Câncer no Brasil. Rio de Janeiro: INCA; 2017.

3. Instituto Nacional de Câncer José Alencar Gomes da Silva. Estimativa 2020: Incidência de câncer no Brasil. Rio de Janeiro: INCA; 2019.

4. Chaimowicz F. Epidemiologia e o envelhecimento no Brasil. In: Freitas EV, PY L. Tratado de geriatria e gerontologia. 3. ed. Rio de Janeiro: Guanabara Koogan; 2011. p. 106-130.

5. Instituto Nacional de Câncer José Alencar Gomes da Silva, Divisão de Apoio à Rede de Atenção Oncoló-

\section{Agradecimentos}

À Fundação de Amparo e Pesquisa à Pesquisa do Estado de Minas Gerais (Fapemig).

\section{Colaboradores}

Buranello MC (0000-0003-1782-1035)* contribuiu para a coleta de dados, análise dos resultados e redação do artigo. Walsh IAP (0000-0002-5684-8612)* contribuiu para a concepção do projeto e revisão do artigo. Pereira GA (0000-0002-9149-6368)* contribuiu para a concepção do projeto, análise estatística dos dados e revisão do artigo. Castro SS (00000002-2661-7899)* contribuiu para a concepção do projeto, redação e revisão do artigo.

gica. Programa Nacional de Controle do Câncer de Mama. Rio de Janeiro: INCA; 2011.

6. Dantas ELR, Lima Sá FH, Carvalho SMF, et al. Genética do Câncer Hereditário. Rev. Bras. Canc. 2009; 55(3):263-269.

7. Agência Nacional de Saúde Suplementar. Nota Técnica 876/2013, Diretriz de Utilização de Genética para os procedimentos de Análise Molecular de DNA e Pesquisa de Microdeleções Microduplicações por FISH, constantes do Anexo II, da Resolução Normativa $n^{\circ}$ 338/2013. Rio de Janeiro: ANSS; 2013.

8. Instituto Nacional de Câncer José Alencar Gomes da Silva. Rede nacional de câncer familial: manual operacional. Rio de Janeiro: INCA; 2009.

9. Instituto Nacional de Câncer José Alencar Gomes da
*Orcid (Open Researcher and Contributor ID). 
Silva. Controle do Câncer de Mama: Documento do Consenso. Rio de Janeiro: INCA; 2004.

10. Ashton-Prolla P, Giacomazzi J, Schmidt AV, et al. Development and validation of a simple questionnaire for the identification of hereditary breast cancer in primary care. BMC Cancer. 2009; (9):283.

11. Instituto Brasileiro de Geografia e Estatística. Censo Demográfico 2010. Famílias e Domicílios. Resultados da amostra. Rio de Janeiro: IBGE; 2012.

12. Organização Mundial da Saúde. Mulheres e saúde: evidências de hoje, agenda de amanhã. Brasília, DF: OMS; 2011.

13. Instituto Nacional de Câncer José Alencar Gomes da Silva. Diretrizes para a detecção precoce do câncer de mama no Brasil. Rio de Janeiro: INCA; 2015.

14. Contador JLS, França EL. Testes não paramétricos para pequenas amostras de variáveis não categorizadas: um estudo. Gestão \& Prod. 2016 [acesso em 2021 jun 4]; 23(03):588-599. Disponível em: https:// doi.org/10.1590/0104-530X357-15.

15. Cloud AJ, Thai A, Liao Y, et al. The impact of cancer prevention guideline adherence on overall mortality in a high-risk cohort of women from the New York site of the Breast Cancer Family Registry. Breast Cancer Res Treat. 2015; 14(9):537-46.

16. Renck DV, Barros F, Domingues MR, et al. Equidade no acesso ao rastreamento mamográfico do câncer de mama com intervenção de mamógrafo móvel no sul do Rio Grande do Sul, Brasil. Cad. Saúde Pública. 2014; 30(1):88-96.

17. Caleffi M, Ribeiro RA, Bedin AJ, et al. Adherence to a Breast Cancer Screening Program and Its Predictors in Underserved Women in Southern Brazil. Cancer Epidemiol Biomarkers Prev. 2010; 19(10):2673-2679.

18. Bellcross CA, Peipins LA, Mccarty FA, et al. Characteristics associated with genetic counseling referral and $\mathrm{BRCAl} / 2$ testing among women in a large integrated health system. Genet Med. 2015; 17(1):43-50.
19. Malone KE, Daling JR, Doody DR, et al. Prevalence and Predictors of BRCAl and BRCA2 Mutations in a Population-Based Study of Breast Cancer in White and Black American Women Ages 35 to 64 Years. Cancer Res. 2006; 66(16):8297-308.

20. Blaes AH, Jewett PI, McKay K, et al. Factors associated with genetic testing in a cohort of breast cancer survivors. Breast J. 2019; (25):1241-1244.

21. Brinton LA, Gierach GL, Pfeiffer RM, et al. Breast Cancer Risk in Older Women: Results from the NIH-AARP Diet and Health Study. Cancer Causes Control. 2014; 25(7):843-857.

22. Lourenco TS, Mauad EC, Vieira RAC. Barreiras no rastreamento do câncer de mama e o papel da enfermagem: revisão integrativa. Rev. Bras. Enferm. 2013; 66(4):585-591.

23. Batiston AP, Tamaki EM, Souza LAS, et al. Conhecimento e prática sobre os fatores de risco para o câncer de mama entre mulheres de 40 a 69 anos. Rev. Bras. Saúde Mater. Infant. 2011; 11(2):163-171.

24. Brum IV, Rodrigues TCGF, Laporte EGJ, et al. Conhecer alguém com câncer de mama influencia a prevalência da adesão ao rastreamento dos cânceres de mama e colo uterino? Rev. Bras. Ginecol Obstet. 2018; 40:203-208.

25. US Preventive Services Task Force. Risk Assessment, Genetic Counseling, and Genetic Testing for BRCA-Related Cancer: US Preventive Services Task Force Recommendation Statement. JAMA. 2019; 322(7):652-665.

26. Vianna FSL, Giacomazzi J, Oliveira Netto CB, et al. Performance of the Gail and Tyrer-Cuzick breast cancer risk assessment models in women screened in a primary care setting with the FHS-7 questionnaire. Genet. Mol. Biol. 2019; 42(1):232-237.

Recebido em 25/08/2020

Aprovado em 10/06/2021

Conflito de interesses: inexistente

Suporte financeiro: Fundação de Amparo à Pesquisa do Estado de Minas Gerais (Fapemig) suporte no 01825-12 